\title{
Clinical utility of capsule endoscopy with flexible spectral imaging color enhancement for diagnosis of small bowel lesions
}

Authors

Institutions
Yasushi Sato' ${ }^{1}$,Tamotsu Sagawa'², Masahiro Hirakawa', Hiroyuki Ohnuma', Takahiro Osuga'1, Yutaka Okagawa', Fumito Tamura', Hiroto Horiguchi' ${ }^{1}$, Kohichi Takada', Tsuyoshi Hayashi', Tsutomu Sato', Koji Miyanishi', Rishu Takimoto ${ }^{1}$, Masayoshi Kobune $^{1}$, Junji Kato ${ }^{1}$

${ }^{1}$ Department of Medical Oncology and Hematology, School of Medicine, Sapporo Medical University, Sapporo, Japan ${ }^{2}$ Division of Gastroenterology, Hokkaido Cancer Center, Sapporo, Japan submitted 24. July 2013 accepted after revision 12. February 2014

\section{Bibliography}

DOI http://dx.doi.org/ 10.1055/s-0034-1365526 Published online: 7.5.2014 Endoscopy International Open 2014; 02: E80-E87

(c) Georg Thieme Verlag KG Stuttgart · New York E-ISSN 2196-9736

\section{Corresponding author:} Vasushi Sato

Department of Medical Oncology and Hematology, School of Medicine, Sapporo Medical University

South 1 West 16, Chuo-ku Sapporo 060-8543

Japan

yasushis@sapmed.ac.jp
Background and study aims: The clinical utility of computed virtual chromoendoscopy with flexible spectral imaging color enhancement (FICE) in capsule endoscopy (CE) remains controversial. To clarify the clinical utility of FICE-enhanced CE in evaluating small bowel lesions, we quantitatively assessed white light (WL), FICE, and blue mode (BM) images and examined the sensitivity of these 3 imaging modes of small-bowel lesions from patients who underwent $\mathrm{CE}$.

Methods: The CIELAB color difference $(\triangle \mathrm{E})$ and visual analogue scales (VAS) were measured in 261 CE images (3 different lesion categories) using WL and FICE set 1,2, and 3, and BM images, respectively. Three endoscopists reviewed CE videos with WL, 3 FICE mode settings, and BM, and compared the sensitivity and detectability for small intestinal diseases from 50 patients who underwent $\mathrm{CE}$.

Results: In the assessment of visibility in the 152 vascular lesion images, the $\triangle \mathrm{E}$ and VAS of FICE set 1,2 , and $B M$ images were significantly higher than that of WL images. In 88 erosion/ulceration

\section{Introduction}

$\nabla$

Capsule endoscopy (CE) is a mainstay for endoscopic examination of small bowel diseases, such as obscure gastrointestinal bleeding (OGIB), Crohn's disease, and polyposis syndromes $[1,2]$. The diagnostic superiority of CE has been proven by studies comparing $\mathrm{CE}$ with other methods for evaluating the small bowel, such as push enteroscopy and radiologic procedures $[3,4]$. CE has been suggested as the first-line modality for diagnosis of OGIB. However, the overall positive diagnostic yield of CE in OGIB is only around 60\% [1]. Recently, to improve diagnostic capability, virtual chromoendoscopy techniques have been proposed to enhance the contrast of microvessels, resolution of superficial mucosal patterns, and color differences [5]. One such modality, the flexible images, the $\triangle \mathrm{E}$ and VAS of FICE set 1 and 2 images were significantly higher than that of WL images. In 21 tumor images, there were no significant differences in $\Delta \mathrm{E}$ among these modalities. When analyzed on a per-patient basis, FICE settings 1 and 2 had the highest sensitivity (100\%) and specificity $(97.3-100 \%)$ for vascular lesions. As for erosive/ ulcerative lesions, FICE setting 2 had the highest sensitivity (100\%) and specificity (97.2\%). For tumors or polyps, WL had the highest sensitivity (90.9\%) and specificity (87.1\%). In per-lesion analysis, FICE settings 1 and 2 showed significantly superior detection ability over WL for vascular lesions. In the detection of erosive/ulcerative lesions, FICE setting 2 was significantly superior to WL. In tumor images, there was no significant improvement with any of the settings relative to $\mathrm{WL}$ images.

Conclusions: FICE is most useful for improving CE image quality and detection in cases of angioectasia and erosion/ulceration of the small intestine.

spectral imaging color enhancement (FICE) system, was developed and introduced in 2005 as a new image processing tool for video endoscopy. FICE is a system that estimates the spectral reflectivity of the target tissue, reconstructs flexible spectral images with different wavelengths calculated from conventional white light (WL) images, and develops new flexible spectral images by selecting and reconstructing RGB wavelengths that emphasize the target [5-8]. FICE is a digital imaging technology that utilizes RAPID software and allows processing of ordinary images captured using standard video capsule devices. Blue mode (BM), an additional image-enhanced modality setting available with the RAPID software package, entails a color coefficient shift of light in the short wavelength range $(490-430 \mathrm{~nm})$ superimposed onto a white light image. The addition of 

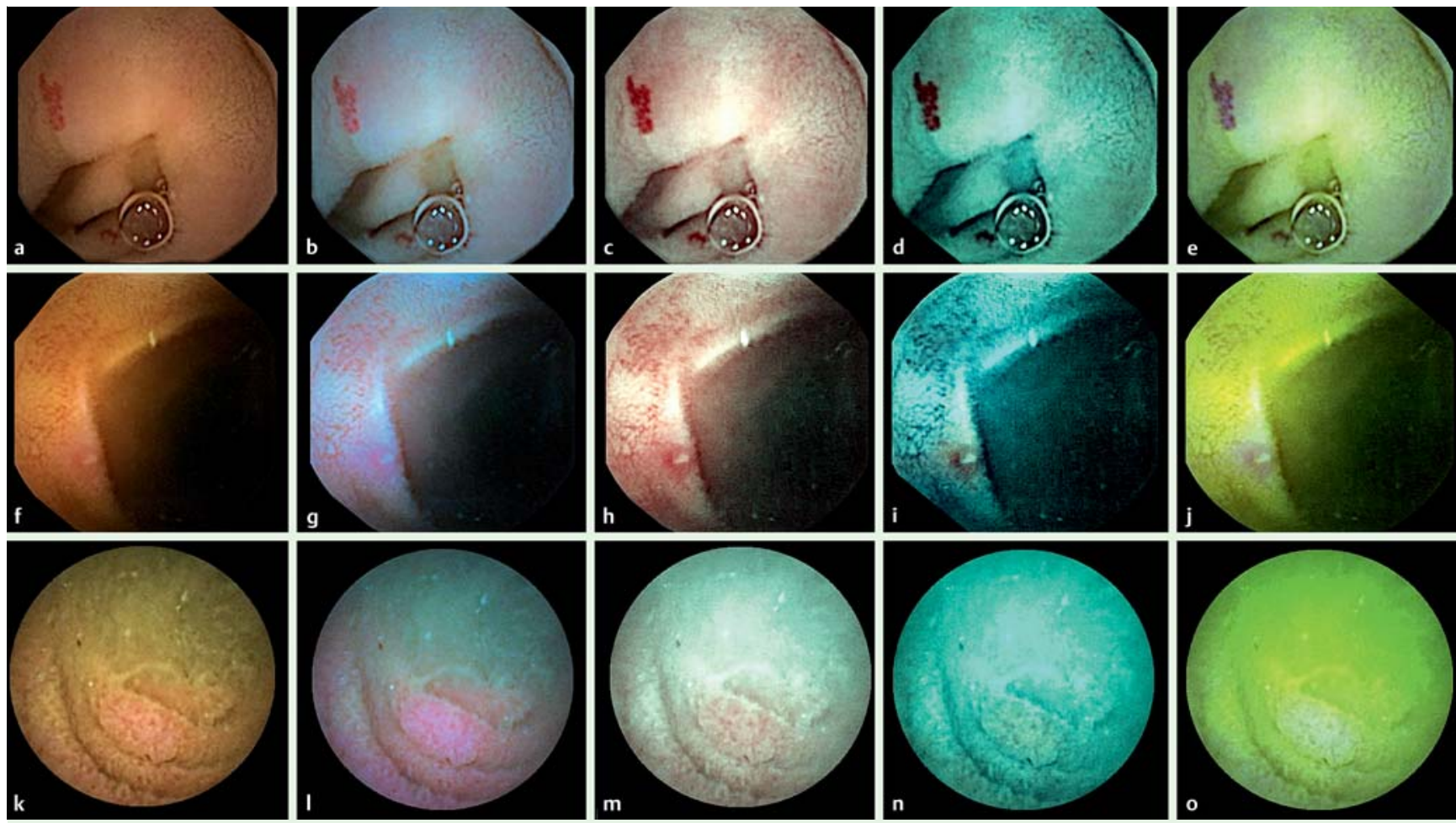

Fig. 1 Capsule endoscopy (CE) images of small intestinal diseases obtained using flexible spectral imaging color enhancement (FICE) and blue mode (BM). Representative images of white light (WL), FICE and BM-CE of a small-bowel angioectasia $(\mathbf{a}-\mathbf{e})$, ulcer ( $\mathbf{f}-\mathrm{j})$, and tumor $(\mathbf{k}-\mathbf{o})$, respectively. WL-CE image $(\mathbf{a}, \mathbf{f}, \mathbf{k})$. BM image $(\mathbf{b}, \mathbf{g}, \mathbf{I})$. FICE-CE images derived from the 3 different wavelength settings (set $1[\mathbf{c}, \mathbf{h}, \mathbf{m}]$, set $2[\mathbf{d}, \mathbf{I}, \mathbf{n}]$, set 3 [e, j, o]).

these technologies to $\mathrm{CE}$ is expected to improve the diagnostic yield. Imagawa and colleagues reported that FICE can improve the visibility of small bowel lesions detected under WL by CE [9]. However, Gupta and associates reported that FICE did not improve the detection of small bowel lesions in comparison with WL [10]. Moreover, Krystallis and coworkers reported that BM offers better image enhancement in CE compared with FICE [11]. Thus, data on the use of FICE and BM in CE are limited and the results are controversial. Therefore, the clinical value of FICE-enhanced CE remains unknown. Studies conducted to date have been limited by the lack of qualitative methods to assess visibility. In this study, we aimed to qualitatively evaluate FICE and BM enhancement in CE, using a RAPID6 Access image diagnostic system, in images of lesions obtained during small bowel $\mathrm{CE}$, and to compare the results with corresponding images obtained using WL. Therefore, to evaluate visibility, we first quantified the color contrast of small bowel lesions using the color space CIELAB method, [12] which associates color perception with colorimetric values, and we used a visual analogue scale (VAS) to assess visibility [13]. We also assessed whether FICE-CE could improve the detection of small-bowel lesions.

\section{Methods}

$\nabla$

\section{CE procedure}

A PillCam SB or SB2 (Given Imaging Ltd, Yoqneam, Israel) was used for CE. All patients included had received $500 \mathrm{ml}$ of polyethylene glycol-based bowel preparation before the examination and were given dimethicone before swallowing the capsule after an overnight fast. A RAPID6 Access CE diagnostic system (Given Imaging Ltd) equipped with the FICE system and BM was used for the analysis. The principle of FICE estimation technology is described elsewhere [5]. FICE set 1 (R, $595 \mathrm{~nm}$; G, $540 \mathrm{~nm}$; and B, $535 \mathrm{~nm})$, FICE set 2 (R, $420 \mathrm{~nm} ; \mathrm{G}, 520 \mathrm{~nm}$; and $B, 530 \mathrm{~nm})$, and FICE set 3 (R, $595 \mathrm{~nm} ; \mathrm{G}, 570 \mathrm{~nm}$; and $B, 415 \mathrm{~nm}$ ), which are the default FICE settings, and BM (wavelength 490-430 nm) were used in this study.

\section{Image interpretation}

To evaluate the visualization of CE images, we retrospectively assessed all images of lesions obtained from 189 patients (123 male, 66 female; median age 53 years) who underwent $\mathrm{CE}$ for small-bowel lesions at Sapporo Medical University Hospital between January 2009 and April 2012.CE was performed for the following reasons: obscure gastrointestinal bleeding (OGIB) $(n=$ 129), examination of the extent of tumor spread ( $n=43)$, chronic abdominal pain or diarrhea $(n=13)$, and miscellaneous $(n=4)$. The 261 lesions in which the final diagnoses were confirmed by balloon enteroscopy, surgery, or periodical observation were chosen on the basis of their overall acceptability as CE images by one of the authors (YS) who has extensive experience with CE. Lesions classified as P0 (no potential for bleeding) were not taken into account [14]. This author did not participate in further evaluation of the images. CE images were categorized according to 3 different lesion categories: i.e., angioectasia $(n=152)$, erosion/ulceration $(n=88)$, or tumor $(n=21)$.

Representative CE images are shown in $\bullet$ Fig. 1 ; the color difference $(\Delta \mathrm{E})$ was examined between each lesion and corresponding background mucosa in WL images, FICE set $1-3$, and BM images. $\triangle \mathrm{E}$ was calculated using the CIE $1976\left(\mathrm{~L}^{*} \mathrm{a}^{*} \mathrm{~b}^{*}\right)$ color space (CIELAB) method.[12] CIE $L^{*} a^{*} b^{*}$ (CIELAB) is a 3-dimensional color space consisting of a black-white axis $\left(L^{*}\right)$, a red-green axis $\left(a^{*}\right)$, and a yellow - blue axis $\left(b^{*}\right)\left(\right.$ Fig. 2 a). $L^{*}$ is defined as light- 
ness, $a^{*}$ as the red - green component, and $b^{*}$ as the yellow-blue component. All endoscopic images were stored in JPEG format. $\triangle \mathrm{E}$ obtained from the WL image, the FICE set 1 , set 2, and set 3 images, and BM images was determined. $\Delta \mathrm{E}$ was measured by a single computer operator (TS) who was able to recognize small bowel lesions and background mucosa in the endoscopic images but had no knowledge of the patients' histories. The operator was initially asked to select 1 sample point at random from a lesion, such as an area of rubefaction, in each WL image, as shown in - Fig. 2 b, and subsequently to select 1 sample point from background mucosa close to the sample points for the small bowel lesion. The corresponding regions were then selected on the FICE and BM images and the color difference was calculated using the method described for WL images ( $\bullet$ Fig. 2 c). One sample point consisted of 81 pixels $(9 \times 9$ pixels $)$ and the median RGB value determined using Adobe Photoshop Elements 2.0. L*a*b* was then calculated based on the median RGB value. The Photoshop RGB value (sRGB value; designated as R', G', and B') indicates "the color dependent on the device." Therefore, an sRGB value does not express an absolute color value and, consequently, it is necessary to convert the SRGB value to the XYZ color system independent of a device. After determining the sRGB values, we calculated $\triangle \mathrm{E}$ and compared differences in $\triangle \mathrm{E}$ among FICE, BM, and WL images.

The VAS was used to score the visibility of small bowel lesions in the images, which were evaluated by 5 expert endoscopists $(\mathrm{MH}$, HO, TO, YO, FT) who had not been informed about the study design. All images were incorporated into a slideshow (Microsoft Office PowerPoint 2008, Microsoft Inc, USA) and displayed on a black background with a 15 -inch screen. For slides containing either WL; FICE set 1, 2, 3 images; or BM images, the assessors were asked to grade the following items on a 100 -mm VAS ( $0=$ low quality; 100 = high quality) [13].

To evaluate the detectability of small bowel lesions in images from each FICE setting or BM compared with the WL image, a total of 50 patients ( 27 males/23 females; median age, 62.5 years) who underwent CE between May 2012 and April 2013 were examined at Sapporo Medical University Hospital. CE was performed for the following reasons: OGIB $(n=34)$, examination of the extent of tumor spread $(n=8)$, chronic abdominal pain or diarrhea $(n=4)$, and miscellaneous $(n=4)$. Three certified endoscopists (YS, TS, MH) who had similar levels of experience in CE image analysis (i.e., more than 100 cases) read the videos in a

Fig.2 Colorimetric evaluation of conventional capsule endoscopy (CE) images and flexible spectral imaging color enhancement (FICE) and blue mode (BM) images. (a) $1976 \mathrm{CIE} L^{*} a^{*} b^{*}$ Space. CIE $L^{*} a^{*} b^{*}$ (CIELAB) is the color space specified by the International Commission on Illumination (Commission Internationale d'Eclairage). The color of images is expressed in terms of 3 coordinate values $\left(L^{*}, a^{*}, b^{*}\right)$, located in a 3-dimensional color space. The 3 coordinates of CIELAB represent the lightness of the color ( $L^{*}=0$ yields black and $L^{*}=100$ indicates diffuse white; specular white may be higher), its position between red and green ( $a^{*}$, negative values indicate green while positive values indicate red), and its position between yellow and blue $\left(b^{*}\right.$, negative values indicate blue and positive values indicate yellow). The color difference ( $\Delta \mathrm{E}$ ) shows the distance between 2 sample regions in the color space. (b) Representative vascular lesion images for calculating color differences. In the area of the yellow square in the conventional image (left), 1 sample point is selected from a vascular lesion and 1 sample point is selected from the background mucosa close to the vascular lesion sample point. The sample points in the corresponding region of FICE setting 2 image (right) were selected using the same method. (c) Protocol for calculating color differences $(\Delta \mathrm{E})$ between small bowel lesions and background mucosa. blinded fashion. In practice, one endoscopist (YS) read videos 1 to 20 with WL, videos 21 to 40 with FICE at settings $1-3$, and videos 41 to 50 with BM. A second reader (TS) read the same sequences of videos using FICE, BM and WL, in that order, and a third reader $(\mathrm{MH})$ read the videos sequentially using $\mathrm{BM}, \mathrm{WL}$, and FICE. The FICE reader read each FICE setting on another day and was blinded to the results. The most relevant findings obtained from CE were documented and classified by each endoscopist as: vascular lesion, erosion/ulceration, tumor, or no abnormality. The numbers of lesions detected and reading times were compared between WL and FICE setting $1-3$, or BM. The final di-
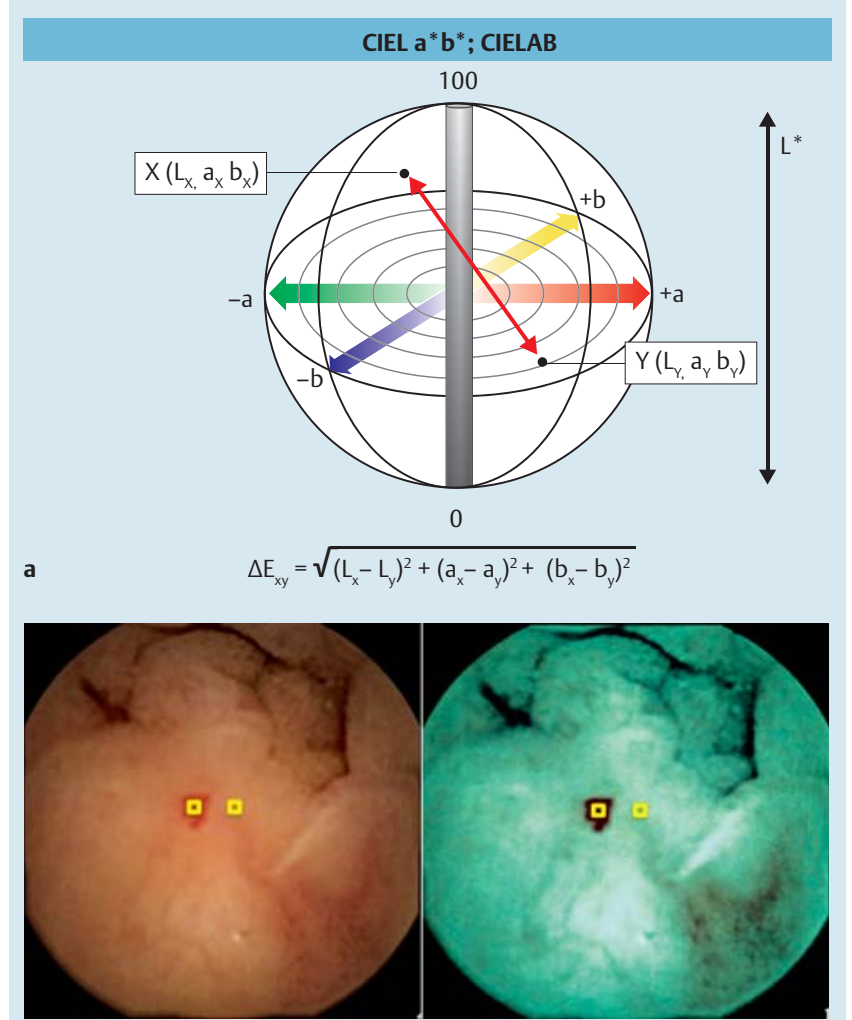

b

1. Standard RGB (sRGB) of the sample (small bowel vascular lesion and background muscosa) was calculated using Adobe Photoshop.

2. Conversion from sRGB to linear RGB. $R=\left(R^{\prime} / 255\right)^{r}$

According to the ITU-R BT.709 standard, a $y$ value of 2.2

3. Conversion from linear RGB to the $X Y Z$ system.

$X=0.4124 \times R+0.3576 \times G+0.1805 \times B$

$Y=0.2126 \times R+0.7152 \times G+0.0722 \times B$

$Z=0.0193 \times R+0.1192 \times G+0.9505 \times B$

4. $L^{*}, a^{*}$, and $b^{*}$ were calculated from $X Y Z$.

The coefficient for the D65 white spot was used:

$X n=95.045 \quad Y n=100 \quad Z n=108.892$

$L^{*}=116 \times f\left(\frac{Y}{Y_{n}}\right)-16$

$a^{*}=500 \times\left\{f\left(\frac{X}{X n}\right)-f\left(\frac{Y}{Y n}\right)\right\}$

$b^{*}=200 \times\left\{f\left(\frac{Y}{Y n}\right)-f\left(\frac{Z}{Z n}\right)\right\}$

5. Color difference $(\Delta \mathrm{E})$ was calculated according to the following formula

$$
\Delta E_{x y}=\sqrt{\left(L_{x}-L_{y}\right)^{2}+\left(a_{x}-a_{y}\right)^{2}+\left(b_{x}-b_{y}\right)^{2}}
$$


agnoses, which were made by several modalities including CE, balloon enteroscopy, surgery and periodical observation, were used as the gold standard for the analyses. This study was approved by our institutional review board. All patients provided written informed consent for participation in the study.

\section{Statistical analysis}

All statistical analyses were performed using StatView version 5.0 software (SAS Institute, Inc., Cary, NC, USA). Parametric data were expressed as mean \pm standard deviation (SD). In all analyses, the mean values were compared by paired Student's $t$-test. A $P$ value less than 0.05 was considered statistically significant.

\section{Results \\ $\nabla$}

Comparison of color differences in WL, FICE, and $\mathrm{BM}$ images quantified using the CIERAB method for each lesion Type

To evaluate the color contrast between 261 small bowel lesion images from 189 patients and their respective background normal mucosa, we determined $\triangle \mathrm{E}$ in WL, FICE set $1-3$, and BM images. The protocols for calculating $\Delta \mathrm{E}$ are shown in $\mathbf{F i g .} \mathbf{2 b}, \mathbf{c}$, and the results for each FICE setting and BM are shown for each lesion type in Table 1. For vascular lesion (152 images), the $\Delta \mathrm{E}$ values were as follows: WL images $=24.3 \pm 9.4$, FICE set $1=76.1 \pm$ 17.4, FICE set $2=78.2 \pm 20.6$, FICE set $3=56.3 \pm 26.2$ and BM set $=$ $67.3 \pm 16.6$. The $\triangle \mathrm{E}$ of the FICE set 1 and 2 , and $\mathrm{BM}$ images was significantly higher than $\Delta \mathrm{E}$ of the WL images $(P<0.01)$. There was no significant difference in $\triangle \mathrm{E}$ between FICE set 1 and 2, BM and FICE set 1, and BM and FICE set 2. For erosion/ulceration (88 images), the $\Delta \mathrm{E}$ values were as follows: $\mathrm{WL}$ images $=18.5 \pm 7.3$, FICE set $1=43.2 \pm 14.9$, FICE set $2=46.9 \pm 17.6$, FICE set $3=31.8 \pm 15.6$, and $\mathrm{BM}=37.2 \pm 14.2$. The $\triangle \mathrm{E}$ of FICE set 1 and 2 images was significantly higher than that of WL images $(P<0.01)$. There was no significant difference in $\triangle \mathrm{E}$ between FICE setting 1 and 2 . For tumors (21 images), the $\Delta \mathrm{E}$ values were as follows: $\mathrm{WL}$ images $=20.2 \pm$ 7.6 , FICE set $1=21.5 \pm 11.2$, FICE set $2=21.2 \pm 8.4$, FICE set $3=23.1$ \pm 9.2 and $B M=23.3 \pm 8.8$. There was no significant difference in $\Delta \mathrm{E}$ among settings. Regarding specific types of tumor, there were 4 lymphangioma, 5 follicular lymphoma, 3 gastrointestinal stromal tumor (GIST), 6 adenomatous polyp, and 3 Peutz-Jeghers polyp cases, and there was no significant difference among settings in the $\Delta \mathrm{E}$ values relative to WL images for the different tumor type settings.
Evaluation of visibility quantified by lesion type using a VAS with WL, FICE, and BM images

We also assessed visibility scores for each of the imaging methods ( Table 1). For vascular lesion (152 images), the VAS scores were as follows: WL images $=50.2 \pm 1.2$, FICE set $1=72.7 \pm 5.2$, FICE set $2=74.0 \pm 14.9$, FICE set $3=58.7 \pm 14.9$, and $\mathrm{BM}=64.8 \pm 4.6$. The VAS scores for FICE set 1 and set 2 and BM images were significantly higher than the score for WL images $(P<0.01)$. However, the score for FICE set 3 was not improved as much as the score for FICE set 1 and set 2, and BM. For erosion/ulceration (88 images), the VAS scores were: $W L$ images $=50.2 \pm 3.0$, FICE set $1=72.9$ \pm 5.4 , FICE set $2=67.9 \pm 5.7$, FICE set $3=53.5 \pm 6.5$, and $B M=59.5 \pm$ 6.5. The VAS scores for FICE set 1 and set 2 were significantly higher than the score for WL images $(P<0.01)$. However, the score for FICE set 3 and BM was not improved as much as the score for FICE set 1 and set 2 . For imaging tumors (30 images), the VAS scores were: $\mathrm{WL}$ images $=50.1 \pm 1.0$, FICE set $1=50.7 \pm 4.4$, FICE set $2=54.0 \pm 3.8$, FICE set $3=50.1 \pm 9.8$, and $\mathrm{BM}=54.1 \pm 5.0$. There was no significant improvement with any of the settings relative to WL images. However, the score for FICE and BM was not decreased relative to the score for WL images.

\section{Sensitivity and specificity of CE for each type of small intestinal disease on a per-patient basis}

A complete examination of the entire small bowel was achieved in $88 \%(44 / 50)$ of cases, with median small bowel transit time being 250 min (range 71-522). The final diagnosis indicated that there were 40 vascular lesions in 12 patients, 42 erosion/ulcerative lesions in 13 patients, and 26 tumors or polyps (follicular lymphoma [ $n=6]$; gastrointestinal stromal tumor $[n=2]$; PeutzJeghers polyps $[\mathrm{n}=2]$; familial adenomatous polyposis $[\mathrm{n}=16]$ ) in 11 patients. The remaining 14 individuals without any significant lesions were also confirmed. The per-patient sensitivity and specificity of CE for the detection of small intestinal diseases are shown in $\bullet$ Table 2 . With respect to vascular lesions, FICE setting 1 and 2 had the highest sensitivity (100\%), and specificity (97.3$100 \%)$. As for erosive/ulcerative lesions, FICE setting 2 had the highest sensitivity (100\%) and specificity (97.2\%). In terms of tumors or polyps, WL had the highest sensitivity (90.9\%) and specificity (87.1\%).

\section{Detection of the number of lesions using FICE or BM imaging enhancement}

The results of WL, FICE, and BM are shown per lesion type in $\bullet$ Table 3. In these 50 patients, a total of 17 angioectasias were identified by WL; 24 were detected by FICE at setting 1, 33 at setting 2, and 18 at setting 3 ; and 20 were detected with BM. There were statistically significant differences between WL and FICE setting

\begin{tabular}{|c|c|c|c|c|c|c|}
\hline \multirow[b]{2}{*}{$\begin{array}{l}\text { CE } \\
\text { setting }\end{array}$} & \multicolumn{2}{|c|}{ Vascular lesion } & \multicolumn{2}{|c|}{ Erosion/ulceration } & \multicolumn{2}{|l|}{ Tumor } \\
\hline & $\Delta \mathrm{E}$ & VAS & $\Delta \mathrm{E}$ & VAS & $\Delta \mathrm{E}$ & VAS \\
\hline WL & $24.3 \pm 9.4$ & $50.2 \pm 1.2$ & $18.5 \pm 7.3$ & $50.2 \pm 3.0$ & $20.2 \pm 7.6$ & $50.1 \pm 1.0$ \\
\hline FICE 1 & $76.1 \pm 17.4^{*}$ & $72.7 \pm 5.2^{*}$ & $43.2 \pm 14.9^{*}$ & $72.9 \pm 5.4^{*}$ & $21.5 \pm 11.2$ & $50.7 \pm 4.4$ \\
\hline FICE 2 & $78.2 \pm 20.6^{*}$ & $74.0 \pm 14.9^{*}$ & $46.9 \pm 17.6^{*}$ & $67.9 \pm 5.7^{*}$ & $21.2 \pm 8.4$ & $54.0 \pm 3.8$ \\
\hline FICE 3 & $56.3 \pm 26.2$ & $58.7 \pm 14.9$ & $31.8 \pm 15.6$ & $53.5 \pm 6.5$ & $23.1 \pm 9.2$ & $50.1 \pm 9.8$ \\
\hline BM & $67.3 \pm 16.6^{*}$ & $64.8 \pm 4.6^{*}$ & $37.2 \pm 14.2$ & $59.5 \pm 6.5$ & $23.3 \pm 8.8$ & $54.1 \pm 5.0$ \\
\hline
\end{tabular}

Abbreviations: BM, blue mode; CE, capsule endoscopy; FICE, flexible spectral imaging color enhancement; VAS, visual analogue scale; WL, white light.

$* P<0.01$ versus White light $C E$ 
Table 2 Per-patient sensitivity and specificity of CE for detecting small intestinal lesions.

\begin{tabular}{|c|c|c|c|c|c|c|c|c|c|c|}
\hline & \multicolumn{5}{|c|}{ \% Sensitivity ( $95 \%$ Cl) } & \multicolumn{5}{|c|}{ \% Specificity ( $95 \%$ Cl) } \\
\hline & WL & FICE 1 & FICE 2 & FICE 3 & BM & WL & FICE 1 & FICE 2 & FICE 3 & BM \\
\hline Vascular & $\begin{array}{l}83.3 \\
(50.8- \\
97.0)\end{array}$ & $\begin{array}{l}100 \\
(69.8- \\
100)\end{array}$ & $\begin{array}{l}100 \\
(69.8- \\
100)\end{array}$ & $\begin{array}{l}75.0 \\
(42.8- \\
93.3)\end{array}$ & $\begin{array}{l}83.3 \\
(50.8- \\
97.0)\end{array}$ & $\begin{array}{l}92.1 \\
(77.5- \\
97.9)\end{array}$ & $\begin{array}{l}100 \\
(88.5- \\
100)\end{array}$ & $\begin{array}{l}97.3 \\
(84.5- \\
99.8)\end{array}$ & $\begin{array}{l}94.7 \\
(80.9- \\
99.0)\end{array}$ & $\begin{array}{l}92.1 \\
(77.5- \\
97.9)\end{array}$ \\
\hline $\begin{array}{l}\text { Erosion/ } \\
\text { Ulceration }\end{array}$ & $\begin{array}{l}84.6 \\
(53.6- \\
97.2)\end{array}$ & $\begin{array}{l}92.3 \\
(62.0- \\
99.5)\end{array}$ & $\begin{array}{l}100 \\
(71.6- \\
100)\end{array}$ & $\begin{array}{l}76.9 \\
(45.9- \\
93.8)\end{array}$ & $\begin{array}{l}84.6 \\
(53.6- \\
97.2)\end{array}$ & $\begin{array}{l}89.2 \\
(73.6- \\
96.4)\end{array}$ & $\begin{array}{l}94.6 \\
(80.4- \\
99.0)\end{array}$ & $\begin{array}{l}97.2 \\
(84.1- \\
99.8)\end{array}$ & $\begin{array}{l}91.9 \\
(76.9- \\
97.8)\end{array}$ & $\begin{array}{l}89.2 \\
(73.6- \\
96.4)\end{array}$ \\
\hline Tumor & $\begin{array}{l}90.9 \\
(57.1- \\
99.5)\end{array}$ & $\begin{array}{l}81.8 \\
(47.7- \\
96.7)\end{array}$ & $\begin{array}{l}81.8 \\
(47.7- \\
96.7)\end{array}$ & $\begin{array}{l}72.7 \\
(39.3- \\
92.6)\end{array}$ & $\begin{array}{l}81.8 \\
(47.7- \\
96.7)\end{array}$ & $\begin{array}{l}87.1 \\
(71.7- \\
95.1)\end{array}$ & $\begin{array}{l}84.6 \\
(68.7- \\
93.5)\end{array}$ & $\begin{array}{l}84.6 \\
(68.7- \\
93.5)\end{array}$ & $\begin{array}{l}84.6 \\
(68.7- \\
93.5)\end{array}$ & $\begin{array}{l}84.6 \\
(68.7- \\
93.5)\end{array}$ \\
\hline
\end{tabular}

BM, blue mode; CE, capsule endoscopy; $\mathrm{Cl}$, confidence interval; FICE, flexible spectral imaging color enhancement; WL, white light.

1 and $2(P=0.02$ and $P=0.003$, respectively). A total of 28 erosive/ ulcerative lesions were detected by WL; 33 by FICE setting 1,41 FICE setting 2, 24 at FICE setting 3 , and 28 with BM. Only FICE setting 2 showed significantly superior detection ability over WL $(P$ $=0.007)$. For tumors, a total of 13 lesions were detected by WL; 13 were detected by FICE setting 1, 14 at FICE setting 2, and 10at FICE setting 3; and 14 were detected by BM. Detection did not differ significantly among groups. With respect to tumor type, 6 adenomatous polyps were detected by $\mathrm{WL}$, and the same adenomatous polyps were detected by FICE setting $1-3$ and BM. Two Peutz-Jeghers polyps were detected by WL and FICE setting 1, 2, and BM, but only 1 was detected by FICE setting 3 . Three follicular lymphoma were detected by WL and by FICE setting 1 ; four were detected by FICE setting 2 and by BM, and 1 was detected by FICE at setting 3 . With each of the imaging settings, 2 GISTs were detected. The analysis times with $\mathrm{WL}(36 \pm 3.9 \mathrm{~min})$ and FICE at the various settings (setting $1,35 \pm 2.9 \mathrm{~min}$; setting $2,37 \pm 3.7 \mathrm{~min}$; setting $3,35 \pm 5.3 \mathrm{~min})$, and $\mathrm{BM}(37 \pm 5.6 \mathrm{~min})$ did not differ significantly.

Positives of FICE imaging and negatives of WL-CE imaging There were 2 of 50 patients (4\%) whose angioectasias were missed with WL-CE imaging and only detected with FICE settings 1 and 2. In these lesions, visualization of the mucosa was impaired by the presence of air bubbles and bile pigments. Representative images are shown in $\bullet$ Fig. $\mathbf{3 a - c}$. There were 2 of 50 patients $(4 \%)$ whose aspirin-induced erosions were missed with WL-CE imaging and only detected with FICE settings 1 and 2. With FICE imaging, it was easier to observe demarcation

Table 3 Number of significant lesions detected by FICE or blue mode imaging enhancement.

\begin{tabular}{|llll|}
$\begin{array}{l}\text { CE } \\
\text { setting }\end{array}$ & $\begin{array}{l}\text { Lesion type } \\
\text { Vascular } \\
\text { lesion }(\boldsymbol{n}=\mathbf{4 0})\end{array}$ & $\begin{array}{l}\text { Erosion/ } \\
\text { ulceration }(\boldsymbol{n}=\mathbf{4 2})\end{array}$ & $\begin{array}{l}\text { Tumor } \\
(\boldsymbol{n}=\mathbf{2 6})\end{array}$ \\
\hline WL & 17 & 28 & 13 \\
\hline FICE 1 & $24^{1}$ & 33 & 13 \\
\hline FICE 2 & $33^{2}$ & $41^{3}$ & 14 \\
\hline FICE 3 & 18 & 24 & 10 \\
\hline BM & 20 & 28 & 14 \\
\hline
\end{tabular}

Abbreviations: BM, blue mode; CE, capsule endoscopy; FICE, flexible spectral imaging color enhancement; WL, white light.

$1 P=0.02$ versus WL-CE

$2 P=0.003$ versus WL-CE

${ }^{3} P=0.007$ versus WL-CE of the lesion compared with WL-CE imaging. Representative images are shown in $\bullet$ Fig. $\mathbf{3 d - f}$.

\section{Discussion}

The diagnostic yield of CE remains unsatisfactory, partly because faint or minute small-bowel lesions can be easily missed. Thus, there is a need for image-enhancement technology to enable visualization of such lesions. However, it remains to be verified which of the available image enhancement modes, such as FICE or BM, is most appropriate for improving the ability to diagnose small-bowel lesions. In fact, conflicting results have been reported to date ( $\bullet$ Table 4). This uncertainty is due in part to the fact that studies conducted so far have been limited by the lack of qualitative methods to assess visibility.

In this study, we demonstrated that FICE-CE provided useful information for the diagnosis of small bowel lesions by enhancing the contrast between lesions and background mucosa. This is the first report to objectively evaluate the quantitative detection capacity of small bowel lesions using the CIELAB method $(\Delta \mathrm{E})$. We found that FICE significantly improved the visibility and detection of small bowel vascular and erosion/ulceration lesions better than the other settings that were evaluated.

FICE uses computerized processing to convert standard RGB signals from the endoscope's charge-coupled device, which represents a type of electronic image-enhanced endoscopy (IEE) [58]. Recent advances in image processing technology have enabled isolation of RGB signals as "image information according to the light at a specific wavelength" [6]. An endoscopic image obtained using light of a specific wavelength is known as a spectral image; such images are obtained by the application of spectral estimation technology. Although spectral images are monochromatic, they are displayed in pseudo-colors by allocating RGB signals in FICE. It is possible to analyze images using a combination of light at different wavelengths in FICE. Therefore, the same degree of brightness can be maintained as in conventional endoscopy, and distant-view observations are possible while maintaining adequate viewing. Obtaining bright images is extremely important in small bowel CE, in which the distance from a lesion cannot arbitrarily be controlled. As a result, FICE-CE would be expected to improve the detection of lesions and their characterization, and as a consequence, may improve the diagnostic yield.

In terms of quantitative contrast enhancement, the color difference $(\Delta E)$ between small bowel vascular or erosion/ulceration lesions and background mucosa was significantly larger with FICE set 1 and 2 images than with WL-CE images; a similar tendency 

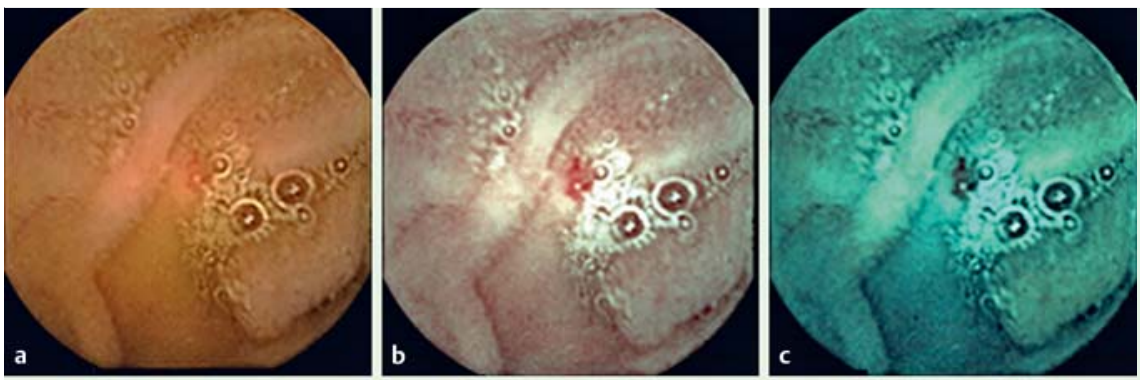

Fig. 3 Two representative cases in which flexible spectral imaging color enhancement (FICE) detected small bowel lesions that were missed with conventional capsule endoscopy imaging. White light (WL) and FICE images of a small-bowel angioectasia with air bubbles and bile pigments $(\mathbf{a}-\mathbf{c})$ and a faint erosion with white spot surrounded by a red halo (d-f). WL-CE image (a,d). FICE-CE setting 1 (b,e), setting 2 (c,f).
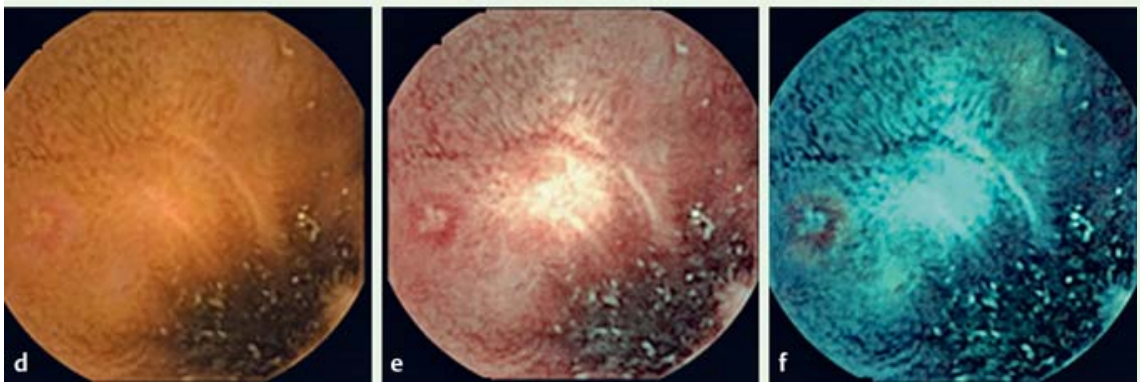

Table 4 Literature on comparison of detectability between WL and each FICE setting.

\begin{tabular}{|c|c|c|c|c|c|c|c|c|c|c|c|}
\hline \multirow[t]{3}{*}{ Reference } & \multirow{3}{*}{$\begin{array}{l}\text { Indication } \\
\text { (number of } \\
\text { patients) }\end{array}$} & \multicolumn{10}{|c|}{ Detectability } \\
\hline & & \multicolumn{3}{|c|}{ Vascular lesion } & \multicolumn{3}{|c|}{ Erosion/ulcer } & \multicolumn{3}{|l|}{ Tumor } & \multirow[t]{2}{*}{ Note } \\
\hline & & FICE 1 & FICE 2 & FICE 3 & FICE 1 & FICE 2 & FICE 3 & FICE 1 & FICE 2 & FICE 3 & \\
\hline $\begin{array}{l}\text { Kobayashi } \\
\text { et al.[19] }\end{array}$ & $\begin{array}{l}\text { OGIB/IDA/ } \\
\text { suspected } \\
\text { tumor/pain } \\
(24)\end{array}$ & $\uparrow$ & $\rightarrow$ & $\rightarrow$ & $\uparrow$ & $\rightarrow$ & $\rightarrow$ & $\downarrow$ & $\rightarrow$ & $\rightarrow$ & $\begin{array}{l}\text { FICE set } 1 \text { missed more } \\
\text { tumors than the WL-CE }\end{array}$ \\
\hline $\begin{array}{l}\text { Matsu- } \\
\text { mura et al. } \\
{[20]}\end{array}$ & $\begin{array}{l}\text { OGIB } \\
(81)\end{array}$ & $\rightarrow$ & & & $\uparrow$ & & & $\rightarrow$ & & & $\begin{array}{l}\text { Diagnostic yield for OGIB } \\
\text { is not improved by FICE }\end{array}$ \\
\hline $\begin{array}{l}\text { Sakai et } \\
\text { al.[15] }\end{array}$ & $\begin{array}{l}\text { OGIB } \\
(12)\end{array}$ & $\uparrow$ & $\uparrow$ & $\rightarrow$ & $\uparrow$ & $\uparrow$ & $\uparrow$ & NA & & & $\begin{array}{l}\text { Detectability of FICE set } 2 \\
\text { was reduced by the pres- } \\
\text { ence of bile-pigments, } \\
\text { but not FICE set } 1\end{array}$ \\
\hline $\begin{array}{l}\text { Nakamura } \\
\text { et al.[16] }\end{array}$ & $\begin{array}{l}\text { Angiodys- } \\
\text { plasia } \\
(50)\end{array}$ & & $\uparrow$ & & & NA & & & NA & & $\begin{array}{l}\text { FICE enables accurate } \\
\text { detection of angiodyspla- } \\
\text { sia in the preview of CE }\end{array}$ \\
\hline $\begin{array}{l}\text { Duque } \\
\text { et al.[17] }\end{array}$ & $\begin{array}{l}\text { OGIB } \\
(20)\end{array}$ & NA & $\uparrow$ & NA & NA & $\uparrow$ & NA & NA & $\rightarrow$ & NA & $\begin{array}{l}\text { FICE set } 2 \text { seems to in- } \\
\text { crease its diagnostic } \\
\text { accuracy of erosions and } \\
\text { angiodysplasias }\end{array}$ \\
\hline $\begin{array}{l}\text { Imagawa } \\
\text { et al.[18] }\end{array}$ & $\begin{array}{l}\text { OGIB/IDA/ } \\
\text { suspected } \\
\text { tumor/pain/ } \\
\text { others } \\
(55)\end{array}$ & $\uparrow$ & $\uparrow$ & $\rightarrow$ & $\rightarrow$ & $\rightarrow$ & $\rightarrow$ & $\rightarrow$ & $\rightarrow$ & $\rightarrow$ & $\begin{array}{l}\text { FICE set } 1 \text { and } 2 \text { are parti- } \\
\text { cularly useful for detect- } \\
\text { ing angioectasias }\end{array}$ \\
\hline $\begin{array}{l}\text { Gupta } \\
\text { et al.[10] }\end{array}$ & $\begin{array}{l}\text { OGIB } \\
(60)\end{array}$ & $\rightarrow$ & NA & NA & $\rightarrow$ & NA & NA & & NA & & $\begin{array}{l}\text { Some vascular lesions } \\
\text { could be more accurately } \\
\text { characterized with FICE } \\
\text { compared with WL-CE }\end{array}$ \\
\hline
\end{tabular}

Abbreviations: $\uparrow$, significantly improved; $\rightarrow$, no significant change; $\downarrow$, significantly decreased; FICE, flexible spectral imaging color enhancement; IDA, iron deficiency anemia; NA, not applicable; OGIB, obscure gastrointestinal bleeding; WL, white light.

was observed with respect to VAS visibility scores. These results indicate that $\triangle \mathrm{E}$ reflects the results of VAS, and thus, that the measurement of $\Delta \mathrm{E}$ would be useful for objectively assessing the visibility of small intestine lesions. The results of the present study are comparable to those of a recent study by Imagawa and colleagues [9], which showed improved visibility for $87.0 \%$ of vascular images analyzed with FICE setting 1 or 2 , and for $53.3 \%$ and $25.5 \%$ of erosion/ulceration images with FICE settings 1 and 2 , respectively.

In our study, there were no significant visibility differences between FICE settings 1 and 2; however, in particular lesions, such as minute small or faint bowel vascular/erosive lesions, the color difference and VAS score were greatest with FICE set 2 (data not shown), which was assumed to be because the image of back- 
ground mucosa of FICE set 2 is blue. It was therefore possible to emphasize the redness of angioectasia or the border of the surrounding reddened area of an erosion/ulceration with inflammatory change more clearly and demarcation of the lesion would be distinct. In contrast, in environments with relatively high amounts of bile juice such as the terminal ileum, FICE set 1 images produced the largest $\Delta \mathrm{E}$ and showed better detectability than FICE set 2 images in cases of vascular or erosion/ulceration lesions (data not shown). This is presumably because bile juice is visualized with almost no color with FICE set 1 , since wavelengths at this setting ( $500 \mathrm{~nm}$ or longer) are not absorbed by bile juice. In fact, Sakai et al. reported that FICE set 1 may reduce the bile-pigment effect and improve the detectability of smallbowel lesions [15]. Additional research is needed to determine which of these settings is most appropriate depending on the condition of the small intestine.

As shown in Table4, there have been conflicting findings to date from studies comparing detectability with WL and each FICE setting, $[10,15-20]$ or instance, Gupta and colleagues reported that FICE did not improve the detection of significant small bowel lesions in comparison with WL [10]. Interestingly, they found that FICE setting 1 provided slightly better characterization of vascular lesions compared with WL. However, in addition to not quantifying visibility, the study did not examine the validity of each FICE settings. It has also been reported that discrepancies in detectability results may be attributable to the lesion location [19], pigment effect [15], conditions of preparation, and experience level of the reader [20]; however, in most of these reports, including our study, FICE settings 1 and/or 2 showed significantly superior ability compared with WL for the detection of vascular and/or erosive/ulcerative lesions. In fact, our main diagnoses were changed by FICE reading versus WL reading in 4 patients $(8 \%)$ with minute vascular lesions and faint erosive lesions. In these cases, FICE can avoid interference from bile juice or residues and enhance the color contrast of the lesions.

Our data showed that FICE-CE is not particularly useful for improving the detection of tumors such as follicular lymphoma, Peutz-Jeghers polyp, and adenomatous polyp, which is consistent with other reports $[9,11]$. In general, it is difficult to demonstrate the usefulness of CE for identifying tumor lesions, since lesions typically vary with respect to morphology, size, surface patterns, hypervascularity, and vascular morphology. In fact, Kobayashi and coworkers [19] reported that FICE set 1 is worse at detecting tumors or polyps than is the WL mode ( Table4). This does not necessarily negate the utility of FICE compared with WL, but suggests that there is a risk of overlooking critical lesions by spectral modifications, in particular, in the exploration of tumor lesions. Therefore, further studies will be needed to assess the ability of FICE-CE to detect specific types of tumor.

$\mathrm{BM}$, which can be obtained by simply enhancing the blue color range of WL images, has been reported to offer better image enhancement in CE compared with FICE, in contrast with the results of our study [11]. However, in that study, FICE was better than BM in image enhancement for vascular lesions such as arteriovenous malformation (AVM). In fact, we found that the detection rate and the sensitivity and specificity for angioectasia cases at FICE settings 1 and 2 were greater than those with BM. In that respect, especially for vascular lesions, it appears that visibility is improved more with FICE than with BM.

Taken together, the data from the present study indicate that FICE setting 1 or 2 was most useful for improving visibility in cases of angioectasia and erosion/ulceration of the small intes- tine. We believe that the enhanced visibility with FICE is responsible for the improved sensitivity, specificity and detection of angioectasias and erosion/ulceration documented in the present study.

In particular, vascular lesions such as angiodysplasias and other vascular abnormalities account for $\sim 80 \%$ of the major source of bleeding from the small bowel [21]; thus, improved detection of angioectasia with FICE would be clinically meaningful for OGIB.

The present study has potential limitations. It was conducted at a single center and the data were analyzed retrospectively. Prospectively assessing the diagnostic yield of CE-FICE and comparison with a comprehensive examination including double-balloon endoscopy would be worth investigating in a future study.

In conclusion, our data show that FICE imaging adds valuable information to conventional $\mathrm{CE}$ imaging and provides better diagnostic ability for small bowel lesions such as angioectasia and erosion/ulceration, especially with the use of FICE setting 1 and/ or 2.A multicenter trial to screen diseases of the small intestine by FICE is warranted to clarify the clinical indications for FICE.

\section{Competing interests: None}

\section{References}

1 Triester SL, Leighton JA, Leontiadis GI et al. A meta-analysis of the yield of capsule endoscopy compared to other diagnostic modalities in patients with obscure gastrointestinal bleeding. Am J Gastroenterol 2005; 100: 2407-2418

2 Wong RF, Tuteja AK, Haslem DS et al. Video capsule endoscopy compared with standard endoscopy for the evaluation of small-bowel polyps in persons with familial adenomatous polyposis (with video). Gastrointest Endosc 2006; 64: 530-537

3 Chong AK, Taylor A, Miller A et al. Capsule endoscopy vs. push enteroscopy and enteroclysis in suspected small-bowel Crohn's disease. Gastrointest Endosc 2005; 61: 255-261

4 Ell C, Remke S, May A et al. The first prospective controlled trial comparing wireless capsule endoscopy with push enteroscopy in chronic gastrointestinal bleeding. Endoscopy 2002; 34: 685-689

5 Pohl J, May A, Rabenstein T et al. Computed virtual chromoendoscopy: a new tool for enhancing tissue surface structures. Endoscopy 2007; 39 : $80-83$

6 Miyake Y, Takeuchi S, Yamataka S. Proceedings of the Thirteenth Color Imaging Conference: Color Science and Engineering Systems, Technologies, and Applications. Arizona: Scottsdale; 2005: 261-263 (ISBN/ ISSN: 0-89208-259-3)

7 Fedeli P, Gasbarrini A, Cammarota G. Spectral endoscopic imaging: the multiband system for enhancing the endoscopic surfacts visualization. J Clin Gastroenterol 2011; 45: 6-15

8 Togashi K, Osawa H, Koinuma $\mathrm{K}$ et al. A comparison of conventional endoscopy, chromoendoscopy, and the optimal-band imaging system for the differentiation of neoplastic and non-neoplastic colonic polyps. Gastrointest Endosc 2009; 69: 734 - 741

9 Imagawa $\mathrm{H}$, Oka S, Tanaka $S$ et al. Improved visibility of lesions of the small intestine via capsule endoscopy with computed virtual chromoendoscopy. Gastrointest Endosc 2011; 73: 299-306

10 Gupta T, Ibrahim M, Deviere J et al. Evaluation of Fujinon intelligent chromo endoscopy-assisted capsule endoscopy in patients with obscure gastroenterology bleeding. World J Gastroenterol 2011; 17: $4590-4595$

11 Krystallis C, Koulaouzidis A, Douglas $S$ et al. Chromoendoscopy in small bowel capsule endoscopy: Blue mode or Fuji Intelligent Colour Enhancement? Dig Liver Dis 2011; 43: 953-957

12 Kuehni RG. Color-tolerance data and the tentative CIE $1976 \mathrm{~L} \mathrm{a} \mathrm{b}$ formula. J Opt Soc Am 1976; 66: 497-500

13 Ghanaati H, Rokni-Yazdi H, Jalali AH et al. Improvement of MR cholangiopancreatography (MRCP) images after black tea consumption. Eur Radiol 2011; 21: 2551-2557

14 Saurin JC, Delvaux M, Gaudin JL et al. Diagnostic value of endoscopic capsule in patients with obscure digestive bleeding: blinded comparison with video push-enteroscopy. Endoscopy 2003; 35: 576-584 
15 Sakai E, Endo H, Kato $S$ et al. Capsule endoscopy with flexible spectral imaging color enhancement reduces the bile pigment effect and improves the detectability of small bowel lesions. BMC Gastroenterol 2012; $12: 83$

16 Nakamura $M$, Ohmiya $N$, Miyahara $R$ et al. Usefulness of flexible spectral imaging color enhancement (FICE) for the detection of angiodysplasia in the preview of capsule endoscopy. Hepatogastroenterology 2012; 59: $1474-1477$

17 Duque G, Almeida N, Figueiredo P et al. Virtual chromoendoscopy can be a useful software tool in capsule endoscopy. Rev Esp Enferm Dig 2012; 104: $231-236$
18 Imagawa H, Oka S, Tanaka S et al. Improved detectability of small-bowel lesions via capsule endoscopy with computed virtual chromoendoscopy: a pilot study. Scand J Gastroenterol 2011; 46: 1133-1137

19 Kobayashi Y, Watabe H, Yamada A et al. Efficacy of flexible spectral imaging color enhancement on the detection of small intestinal diseases by capsule endoscopy. J Dig Dis 2012; 13: 614-620

20 Matsumura T, Arai $M$, Sato $T$ et al. Efficacy of computed image modification of capsule endoscopy in patients with obscure gastrointestinal bleeding. World J Gastrointest Endosc 2012; 4: 421-428

21 Raju GS, Gerson L, Das A et al. American Gastroenterological Association (AGA) Institute medical position statement on obscure gastrointestinal bleeding. Gastroenterology 2007; 133: 1694-1696 